\title{
$1.3 \mathrm{~kW}$ monolithic linearly-polarized single-mode MOPA and strategies for mitigating mode instabilities
}

\author{
Rumao Tao, Pengfei Ma, Xiaolin Wang ${ }^{*}$, Pu Zhou ${ }^{* *}$, Zejin Liu \\ College of Optoelectronic Science and Engineering, National University of Defense Technology, Changsha 410073, \\ China \\ "chinawxllin@163.com; ${ }^{* *}$ zhoupu203@163.com
}

\begin{abstract}
We report on the high power amplification of 1064nm linearlypolarized laser in all-fiber polarization-maintained MOPA, which can operate at output power level of $1.3 \mathrm{~kW}$. The main amplifier was pumped with six $915 \mathrm{~nm}$ laser diodes, and the slope efficiency is $65.3 \%$. The beam quality $\left(M^{2}\right)$ was measured to be $<1.2$ at full power operation. The polarization extinction rate of the fiber amplifier was measured to be above 94\% before mode instabilities (MI) sets in, which reduced to about $90 \%$ after the onset of MI. Power scaling capability of strategies for suppressing MI is analyzed based on a novel semi-analytical model, the theoretical results of which agree with the experimental results. It shows that mitigating MI by coiling the gain fiber is an effective and practical way in standard double-cladding large mode area fiber, and, by tight coiling of the gain fiber to the radius of $5.5 \mathrm{~cm}$, the MI threshold can be increased to 3 times higher than that without coiling or loose coiling. Experimental study has been carried out to verify the idea, which has proved that MI was suppressed successfully in the amplifier by tight coiling.
\end{abstract}

(C)2014 Optical Society of America

OCIS codes: (000.0000) General; (000.2700) General science.

\section{References and links}

1. B. Samson and A. Carter, "Recent Progress on Power Scaling Narrow Linewidth Fiber Amplifiers and Their Applications," The Review of Laser Engineering 41, 714-717 (2010)

2. A. Carter, J. Edgecumbe, D. P. Machewirth, J. Galipeau, B. Samson, K. Tankala and M. O'Connor, "Recent progress in the development of kW-level monolithic PM-LMA fiber amplifiers," [待查]

3. S. Belke, F. Becker, B. Neumann, S. Ruppik, U. Hefter, "Completely monolithic linearly polarized high-power fiber laser oscillator," Proc. of SPIE 8961, 896124 (2014).

4. B. samson, A. Carter and K. Tankala, "rare-earth fibres power up," Nature Phtonics 5, 466-467 (2011).

5. K. Brar, M. Savage-Leuchs, J. Henrie, S. Courtney, C. Dilley, R. Afzal and E. Honea, "Threshold power and fiber degradation induced modal instabilities in high power fiber amplifiers based on large mode area fibers," Proc. of SPIE 8961, 89611R (2014).

6. C.Wirth, T.Schreiber, M.Rekas, I.Tsybin, T.Peschel, R.Eberhardt, and A.Tünnermann, "High-power linearpolarized narrow linewidth photonic crystal fiber amplifier," Proc. SPIE 7580, 75801H (2010).

7. C. Robin, I. Dajani, C. Zeringue, B. Ward, and A. Lanari, "Gain-tailored SBS suppressing photonic crystal fibers for high power applications," Proc. of SPIE 8237, 82371D (2012).

8. M. Karow, H. Tunnermann, J. Neumann, D. Kracht and P. Wessels, "Beam quality degradation of a singlefrequency $\mathrm{Yb}$-doped photonic crystal fiber amplifier with low mode instability threshold power", Opt. Lett. 37, 4242-4244 (2012).

9. B. Ward, C. Robin, and I. Dajani, "Origin of thermal modal instabilities in large mode area fiber amplifiers," Opt. Express 20, 11407-11422 (2012).

10. N. Haarlammert, O. de Vries, A. Liem, A. Kliner, T. Peschel, T. Schreiber, R. Eberhardt, and A. Tünnermann, "Build up and decay of mode instabilityin a high power fiber amplifier," Opt. Express 20, 13274-13283 (2012).

11. R. Tao, P. Ma, X. Wang, P. Zhou, and Z. Liu, "Experimental study on mode instabilities in all-fiberized highpower fiber amplifiers," Chin. Opt. Lett. 12(Suppl.), S20603 (2014). 
12. X. Wang, R. Tao, H. Xiao, P. Zhou, C. Zhang and X. Xu, "Experimental study of mode instability and thermal induced effects in all-fiber amplifier," presented in Advanced Solid State Lasers, Paris France, paper JTh2A.44 (2013).

13. A. Smith and J. Smith, "Mode instability in high power fiber amplifiers," Opt. Express 19, 10180-10192 (2011).

14. K. R. Hansen, T. T. Alkeskjold, J. Broeng, and J. Lægsgaard, "Theoretical analysis of mode instability in highpower fiber amplifiers," Opt. Express 21, 1944-1971 (2013).

15. L. Dong, "Stimulated thermal Rayleigh scattering in optical fibers", Opt. Express 21, 2642-2656 (2013).

16. C. Jauregui, H. Otto, F. Stutzki, F. Jansen, J. Limpert, and A. Tünnermann, "Passive mitigation strategies for mode instabilities in high-power fiber laser systems," Opt. Express 21, 19375-19386 (2013).

17. A. V. Smith and J. J. Smith, "Maximizing the mode instability threshold of a fiber amplifier,” arXiv:1301.3489 [physics.optics] (2013).

18. H.-J. Otto, C. Jauregui, F. Stutzki,F. Jansen, J. Limpert, and A. Tünnermann, "Controlling mode instabilities by dynamic mode excitation with an acousto-optic deflector," Opt. Express 21, 17285-17298 (2013).

19. H.-J.Otto, F.Stutzki, F.Jansen, T.Eidam, C.Jauregui, J.Limpert, and A.Tünnermann, "Temporal dynamics of mode instabilities in high-power fiber lasers and amplifiers," Opt. Express 20, 15710-15722 (2012).

20. Rumao Tao, Pengfei Ma, Xiaolin Wang, Pu Zhou, Zejin Liu, "Study of Mode Instabilities in High Power Fiber Amplifiers by Detecting Scattering Light," presented in International Photonics and OptoElectronics Meetings, Wuhan, paper FTh2F.2 (2014).

21. M. Laurila, M. M. Jørgensen, K.R. Hansen, T. T. Alkeskjold, J. Broeng, and J. Lægsgaard, "Distributed mode filtering rod fiber amplifier delivering 292W with improved mode stability," Opt. Express 20, 5742-5753 (2012).

22. M. N. Ozisik. Heat Conduction, 2nd Edition. John Wiley \& Sons, Inc., New York (1993).

23. M. M. Jørgensen, K. R. Hansen, M. Laurila, T. T. Alkeskjold, J. Lægsgaard, "Modal instability of rod fiber amplifiers: a semi-analytic approach,” Proc. of SPIE 8601, 860123 (2013)

24. M. M. Jørgensen, K. R. Hansen, M. Laurila, T. T. Alkeskjold, J. Lægsgaard, "Fiber amplifiers under thermal loads leading to transverse mode instability," Proc. of SPIE 8961, 89612P (2014).

25. C. Spiegelberg, J. Geng, Y. Hu, Y. Kaneda, S. Jiang, N. Peyghambarian, "Low-noise narrow-linewidth fiber laser at 1550nm," Journal of Lightwave Technology 22, 57-62 (2003).

26. A. V. Smith and J. J. Smith, "Increasing mode instability thresholds of fiber amplifiers by gain saturation," Opt. Express 21, 15168-15182 (2013).

27. J.P. Koplow, D.A.V. Kliner, and L. Goldberg, "Single mode operation of a coiled multi-mode fiber amplifier", Opt. Letters, 25, 442-444 (2000)

28. K. Hejaz, A. Norouzey, R. Poozesh, A. Heidariazar, A. Roohforouz, R. Rezaei Nasirabad, N. Tabatabaei Jafari , A. Hamedani Golshan, A. Babazadeh and M. Lafouti, "Controlling mode instability in a $500 \mathrm{~W}$ ytterbium-doped fiber laser," Laser Phys. 24, 025102 (2014).

29. D. Marcuse, "Curvature loss formula for optical fibers,” J. Opt. Soc. Am.66, 216-220 (1976).

30. M. L. Stock, C.-H. Liu, A. Kuznetsov, G. Tudury, A. Galvanauskas, T. Sosnowski, "Polarized, 100 kW peak power, high brightness nanosecond lasers based on 3C optical fiber", Proc. of the SPIE 7914, 79140U (2011).

31. Tsai-wei Wu, Liang Dong and Herbert Winful, "Bend performance of leakage channel fibers," Opt. Express 16, 4278-4285(2008)

32. Liang Dong, Kunimasa Saitoh, Fanting Kong, PaulFoy, Thomas Hawkins, Devon Mcclane, and Guancheng Gu, “All-Solid Photonic Bandgap Fibers for High Power Lasers (Invited Paper)," Proc. of SPIE 8547, 85470J (2012).

\section{Introduction}

Many applications, such as coherent lidar system, nonlinear frequency conversion, coherent beam combining architectures, require high power linearly-polarized laser sources with single mode operation or near-diffraction-limited beam quality [1,2]. Recently, linearly-polarized fiber laser with $1 \mathrm{~kW}$ output power has been achieved in a monolithic fiber Bragg grating (FBG)-based Fabry-Pérot cavity [3], which employed a pairs of high power FBGs. It is a technological challenge to design FBG that can withstand multi-kilowatt power, and further power scaling may confront with some technological difficulties. Fiber laser systems based on MOPAs are typically capable of reaching high output powers, while also offering more flexibility in terms of linewidth and polarization control than a simple grating based laser [4]. Most of the high power fiber laser systems with random polarized output are based on MOPA at the moment, which has achieved output power as high as tens of kilowatt. However, power scaling of linearly-polarized MOPAs to multi-kilowatt level is currently limited by the onset of mode instabilities (MI) [4-6]. Although lot of work has been carried out to deal with MI experimentally and theoretically [7-19], few methods to mitigate MI effectively in all-fiber MOPA configuration with standard step-index large mode area (LMA) fiber have been 
proposed, and MI-free power scaling in all-fiber MOPA, which are based on standard stepindex PM LMA fibers, is even more challenging.

In this manuscript, we present a 1.3kW-level all-fiber Yb-doped PM fiber amplifier with linearly-polarized single-mode operation. We also discuss experiments, coupled with numerical modeling, to estimate the further power scaling capability of various strategies to mitigate MI. Numerical modeling results suggest that MI-free single-mode output powers in excess of $3 \mathrm{~kW}$ could be realized in standard step-index LMA fiber.

\section{Experimental setup and results}

A monolithic, all-fiber, Yb-doped PM fiber amplifier is shown in Fig. 1. The seed laser in the experiment is a $50 \mathrm{~mW}$ linearly-polarized continous-wave laser with central wavelength at $\sim 1064 \mathrm{~nm}$, which was then amplified in two pre-amplifier stages to $\sim 25 \mathrm{~W}$. The main amplifier consists of a $20 \mathrm{~m}$ PM double-clad LMA Yb-doped fiber (YDF) with $21 \mu \mathrm{m}$ diameter/0.064 NA core and $400 \mu \mathrm{m}$ diameter/0.44 NA cladding, which is coiled loosely. Six multimode fiber-pigtailed $915 \mathrm{~nm}$ laser diodes were used to pump the gain fiber through a $(6+1) \times 1$ signal/pump combiner, which can provide a maximum pump power of about $2 \mathrm{~kW}$. Approximate $0.75 \mathrm{~m}$ long passive fiber is spliced to the gain fiber for power delivery, the output end of which is angle cleaved in order to prevent parasitic feedback from Fresnel reflection.

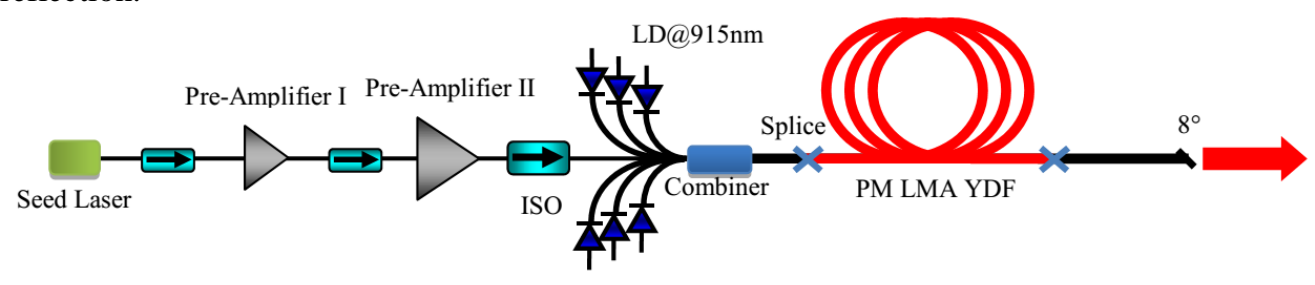

Fig. 1. The architecture of the all-fiber PM amplifier

The achieved output power at different pump power levels is shown in Fig. 2(a), which is measured after the output beam passed through a dichroic mirror. The slope efficiency of the amplifier is $65.3 \%$ with respect to launched pump power, and the pump-limited maximum output power is $1261 \mathrm{~W}$. The inset picture in Fig. 2(a) shows the measured far field beam profile at the maximal operation power, and the beam quality factor $M^{2}$ is measured by $M^{2}$ 200s (Spiricon) to be $<1.2$ in both directions, which indicates the single mode operation of the main amplifier. Fig. 2(b) shows the measured spectra (by an optical spectrum analyzer, AQ6370C Yokogawa) at full power operation, which shows that a high SNR has been achieved and no signs of parasitic lasing or significant levels of amplified spontaneous emission. Fig. 2(c) shows the measurement of polarization extinction rate (PER) for different pump power levels. It can be seen that PER is above $90 \%$ in the whole range, which indicates a linearly-polarized operation. The sudden degradation from $94 \%$ to $90 \%$ above $1.2 \mathrm{~kW}$ was caused by MI while the slowly degradation of PER as lasing power increases may be caused by the temperature increase of the fiber.
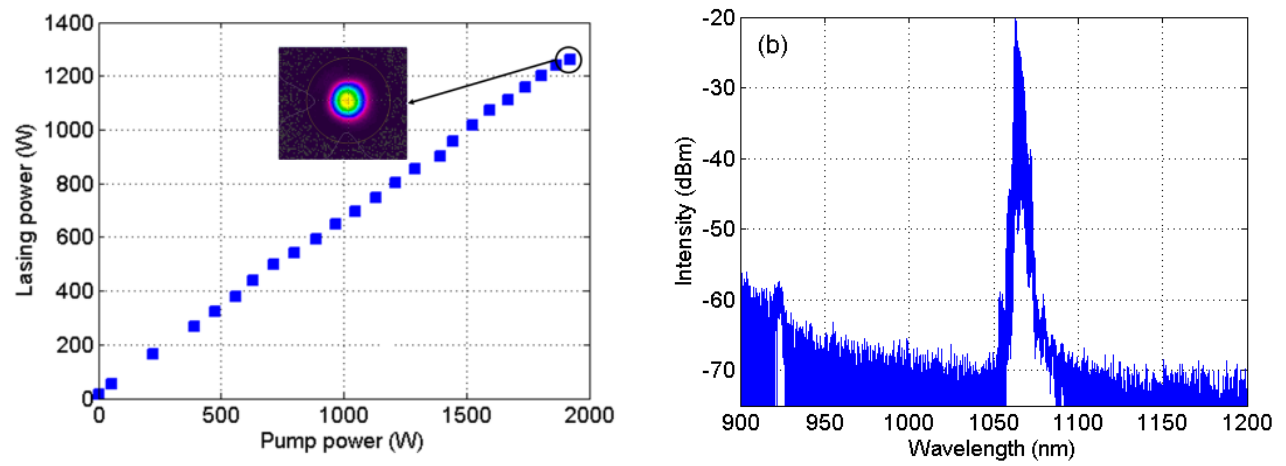


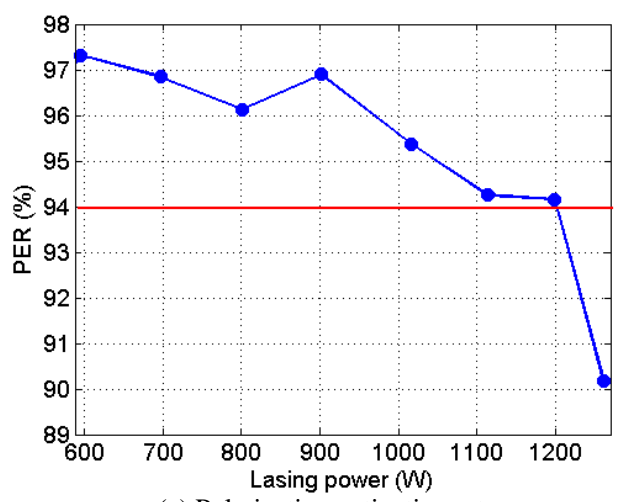

(c) Polarization extinction rate.

Fig. 2. Output characteristics of the main amplifier.

An InGaAs photo detector (150 MHz, $700-1800 \mathrm{~nm}$, Thorlabs) with a pinhole of $1.5 \mathrm{~mm}$ diameter was put in the center of the collimated beam to monitor the onset of MI $[10,11,19]$. Time traces at different output power are shown in Fig. 3(a), and the DC component of the electrical signal is removed. From Fig. 3 (a), we can obtain that although the output power is still pump limited to $1.26 \mathrm{~kW}$, we are operating at the MI threshold (@1.26kW). Although the MI has set in, deterioration of beam quality has not been observed (Fig. 2(a)), which is due to that the fraction of high order mode (HOM) is relatively small at the beginning of MI [18]. It also shows that, at the onset of MI, the amplitude of the time trace is near the same as that without MI in the time period T1 and become higher than that without MI in time period T2. Applying Fourier analysis on the time traces to calculate the corresponding Fourier spectra, we obtained the frequency distribution of the beam fluctuation as shown in Fig. 3(b). The frequency components of the beam fluctuations distributed in the range of $0 \sim 200 \mathrm{~Hz}$ at power of $1.14 \mathrm{~kW}$, which means that the output beam profile is stable; however, further increasing the output power, frequency components in $0 \sim 3 \mathrm{kHz}$ showed up as well as that background noise increases, which indicated the sign of instable beam profile appeared and that the amplifier are approaching the threshold MI. Similar to the observation in [20], the instability of MI has a grown-process: at the start (T1), only background noise increase, which means stable beam profile and indicate that MI may relate to noise [14]; after a few seconds (T2), frequency components shown up, which indicates unstable beam profile. After multiple power cycles near the maximum power, we observed that the threshold reduced to below $1.2 \mathrm{~kW}$, which may due to the fiber degradation $[5,21]$.

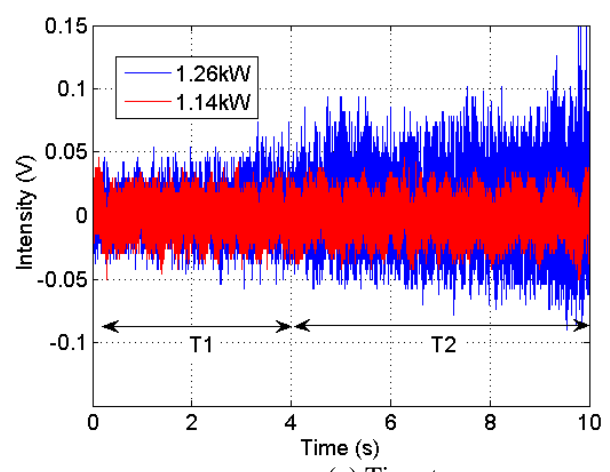

(a) Time traces

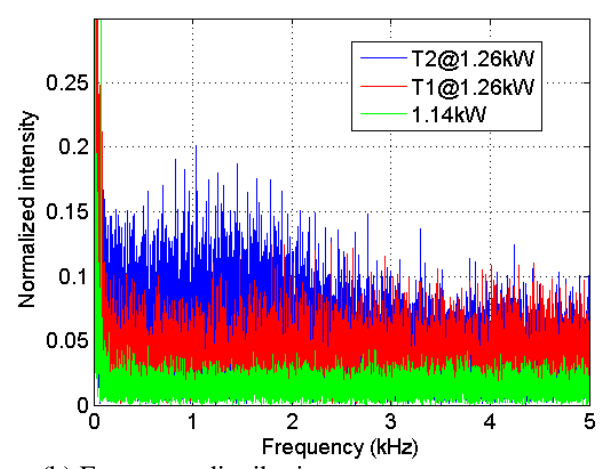

(b) Frequency distribution

Fig. 3. Fluctuation characteristics of the output beam. 


\section{Strategies for suppressing mode instabilities}

\subsection{Theoretical model}

As shown in the previous section, the linearly-polarized single-mode output of fiber laser is limited by the onset ot MI. Work should be carried out to study the further power scaling of the amplifier with single-mode linearly-polarized output. One way is to carry out numerical study on MI and to find way to mitigate this phenomenon effectively. In high power fiber laser systems, most of the fibers are weakly guided fibers, where optical fields can be well approximated by linearly polarized (LP) modes. For linearly-polarized fiber lasers, the optical field of the signal propagating in the fiber is expressed in the conventional LP mode representations

$$
E(r, \phi, z, t)=\sum_{m=0}^{\infty} \sum_{n=1}^{\infty} A_{m n}(z, t) \psi_{m n}(r, \phi) e^{j\left(\beta_{m n} z-\omega_{m n} t\right)}+c . c .
$$

where $m$ and $n$ is azimuthal and radial mode numbers respectively. $A_{m n}(z, t), \beta_{m n}$, and $\psi_{m n}(r, \phi)$ are slowly varying mode amplitudes, propagation constants, and normalized mode profiles of LPmn mode. Assuming the case that the fiber amplifiers are operating below or near the MI threshold, we therefore include only the fundamental mode $\left(\mathrm{LP}_{01}\right)$ and one of the two degenerate $\mathrm{LP}_{11}$ modes and the subscripts of 01 and 11 are replaced with 1 and 2 for $\mathrm{LP}_{01}$ mode and $\mathrm{LP}_{11}$ mode, respectively. Then the signal intensity $I_{\mathrm{s}}$ can be written as

$$
\begin{aligned}
I_{s}(r, \phi, z, t) & =2 n_{0} \varepsilon_{0} c E(r, \phi, z, t) E(r, \phi, z, t)^{*} \\
& =I_{0}+\tilde{I}
\end{aligned}
$$

with

$$
\begin{gathered}
I_{0}=I_{11}(z, t) \psi_{1}(r, \phi) \psi_{1}(r, \phi)+I_{22}(z, t) \psi_{2}(r, \phi) \psi_{2}(r, \phi) \\
\tilde{I}=I_{12}(z, t) \psi_{1}(r, \phi) \psi_{2}(r, \phi) e^{j(q z-\Omega t)}+I_{21}(z, t) \psi_{1}(r, \phi) \psi_{2}(r, \phi) e^{-j(q z-\Omega t)} \\
I_{k l}(z, t)=4 n_{0} \varepsilon_{0} c A_{k}(z, t) A_{t}^{*}(z, t) \\
q=\beta_{1}-\beta_{2}, \Omega=\omega_{1}-\omega_{2}
\end{gathered}
$$
by

The temperature distribution is governed by heat transportation equation, which is given

$$
\nabla^{2} T(r, \phi, z, t)+\frac{Q(r, \phi, z, t)}{\kappa}=\frac{1}{\alpha} \frac{\partial T(r, \phi, z, t)}{\partial t}
$$

where $\alpha=\kappa / \rho C, \rho$ is the density, $C$ is the specific heat capacity, and $\kappa$ is the thermal conductivity. Since the heat in high power fiber amplifiers is mainly generated from the quantum defect and absorption, the volume heat-generation density $Q$ can be approximately expressed as

$$
Q(r, \phi, z, t) \cong g(r, \phi, z, t)\left(\frac{v_{p}-v_{s}}{v_{s}}\right) I_{s}(r, \phi, z, t)
$$

and $g(r, \phi, z, t)$ is the gain of the amplifier

$$
g(r, \phi, z, t)=\left[\left(\sigma_{s}^{a}+\sigma_{s}^{e}\right) n_{u}(r, \phi, z, t)-\sigma_{s}^{a}\right] N_{Y b}(r, \phi)
$$

where $v_{\mathrm{p}(\mathrm{s})}$ is the optical frequencies, $\sigma_{s}^{a}$ and $\sigma_{s}^{e}$ are the signal absorption and emission cross sections, $\sigma_{p}^{a}$ and $\sigma_{p}^{e}$ are the pump absorption and emission cross sections, $N_{Y b}(r, \phi)$ is the doping profile, the population inversion $n_{\mu}$ is given in [13].

Assume that the fiber is water cooled, the appropriate boundary condition for the heat equation at the fiber surface is

$$
\kappa \frac{\partial T}{\partial r}+h_{q} T=0
$$


where $h_{\mathrm{q}}$ is the convection coefficient for the cooling fluid. By adopting the integral-transform technique to separate variables in the cylindrical system [22], Eq. (4), combined with Eqs. (5) and (6), can be solved as

$$
\begin{aligned}
& T(r, \phi, z, t)=\frac{1}{\pi} \frac{\alpha n_{2}}{\eta} \sum_{v} \sum_{m=1}^{\infty} \frac{R_{v}\left(\delta_{m}, r\right)}{N\left(\delta_{m}\right)} \\
& \times \int_{t^{\prime}=0}^{t}\left[\begin{array}{l}
B_{11}(\phi, z) I_{11}\left(z, t^{\prime}\right)+B_{22}(\phi, z) I_{22}\left(z, t^{\prime}\right) \\
+B_{12}(\phi, z) I_{12}\left(z, t^{\prime}\right) e^{j\left(q z-\Omega t^{\prime}\right)}+B_{12}(\phi, z) I_{12}^{*}\left(z, t^{\prime}\right) e^{-j\left(q z-\Omega t^{\prime}\right)}
\end{array}\right] e^{-\alpha \delta_{m}^{2}\left(t-t^{\prime}\right)} d t^{\prime}
\end{aligned}
$$

with

$$
\begin{gathered}
B_{k 1}(\phi, z) \\
=\left\{\begin{array}{c}
\int_{0}^{2 \pi} d \phi^{\prime} \int_{0}^{R} g_{0} R_{v}\left(\delta_{m}, r^{\prime}\right) \cos v\left(\phi-\phi^{\prime}\right) \frac{\psi_{k}\left(r^{\prime}, \phi^{\prime}\right) \psi_{k}\left(r^{\prime}, \phi^{\prime}\right)}{1+I_{0} / I_{\text {saturation }}} d r^{\prime}, \quad k=l \\
\int_{0}^{2 \pi} d \phi^{\prime} \int_{0}^{R} g_{0} R_{v}\left(\delta_{m}, r^{\prime}\right) \cos v\left(\phi-\phi^{\prime}\right) \frac{\psi_{k}\left(r^{\prime}, \phi^{\prime}\right) \psi_{l}\left(r^{\prime}, \phi^{\prime}\right)}{\left(1+I_{0} / I_{\text {saturation }}\right)^{2}} d r^{\prime}, k \neq l
\end{array}\right. \\
N\left(\delta_{m}\right)=\int_{0}^{R} r R_{v}^{2}\left(\delta_{m}, r\right) d r, n_{2}=\frac{\eta}{\kappa}\left(\frac{v_{p}-v_{s}}{v_{s}}\right)
\end{gathered}
$$

where $v=0,1,2,3 \ldots$ and repalce $\pi$ by $2 \pi$ for $v=0, \eta$ is the thermal-optic coefficient, $R$ is the radius of the inner cladding, $g_{0}$ is the small signal gain and $I_{\text {saturation }}$ is the saturation intensity. $R_{v}\left(\delta_{m}, r\right)$ is $h$ given by $R_{v}\left(\delta_{m}, r\right)=J_{v}\left(\delta_{m} r\right)$ and $\delta_{m}$ is the positive roots of $\delta_{m} J_{v}{ }^{\prime}\left(\delta_{m} R\right)+\frac{h_{q}}{J_{v}}\left(\delta_{m} R\right)=0$. Considering effective refractive index of gain from amplifier, the total refrackive index, which attributes to gain $\left(n_{g} \leq n_{0}\right)$ and nonlinearity $\left(n_{N L} \leq n_{0}\right)$, can be expressed as

$$
n^{2}=\left(n_{0}+n_{g}+n_{N L}\right)^{2} \cong n_{0}^{2}-j \frac{g(r, \phi, z, t) n_{0}}{k_{0}}+2 n_{0} n_{N L}
$$

where $n_{N L}$ is given by

$$
\begin{aligned}
& n_{N L}(r, \phi, z, t) \\
= & \eta T(r, \phi, z, t) \\
= & h_{11}(r, \phi, z, t)+h_{22}(r, \phi, z, t)+h_{12}(r, \phi, z, t) e^{j q z}+h_{21}(r, \phi, z, t) e^{-j q z}
\end{aligned}
$$

with

$$
h_{k l}(r, \phi, z, t)=\left\{\begin{array}{c}
\frac{\alpha n_{2}}{\pi} \sum_{v} \sum_{m=1}^{\infty} \frac{R_{v}\left(\delta_{m}, r\right)}{N\left(\delta_{m}\right)} \int_{0}^{t} B_{k k}(\phi, z) I_{k k}\left(z, t^{\prime}\right) e^{-\alpha \delta_{m}^{2}\left(t-t^{\prime}\right)} d t^{\prime}, k=l \\
\frac{\alpha n_{2}}{\pi} \sum_{v} \sum_{m=1}^{\infty} \frac{R_{v}\left(\delta_{m}, r\right)}{N\left(\delta_{m}\right)} \int_{0}^{t} B_{k l}(\phi, z) I_{k l}\left(z, t^{\prime}\right) e^{-\alpha \delta_{m}^{2}\left(t-t^{\prime}\right)-j \Omega t^{\prime}} d t^{\prime}, k \neq l
\end{array}\right.
$$

Inserting Eqs. (1) and (10) into the wave equation, after very tedious but straightforward derivations, we have obtained the coupled-mode equations

$$
\begin{gathered}
\frac{\partial\left|A_{1}\right|^{2}}{\partial z}=\iint g(r, \phi, z) \psi_{1} \psi_{1} r d r d \phi\left|A_{1}\right|^{2} \\
\frac{\partial\left|A_{2}\right|^{2}}{\partial z}=\left[\iint g(r, \phi, z) \psi_{2} \psi_{2} r d r d \phi+\left|A_{1}\right|^{2} \chi(\Omega, t)\right]\left|A_{2}\right|^{2}
\end{gathered}
$$

with

$$
\chi(\Omega)=2 \frac{n_{0} \omega_{2}^{2}}{c^{2} \beta_{2}} \operatorname{Im}\left(4 n_{0} \varepsilon_{0} c \iint \bar{h}_{12} \psi_{1} \psi_{2} r d r d \phi\right)
$$




$$
\bar{h}_{k l}(r, \phi, z)=\frac{\alpha n_{2}}{\pi} \sum_{v} \sum_{m=1}^{\infty} \frac{R_{v}\left(\delta_{m}, r\right)}{N\left(\delta_{m}\right)} \frac{B_{k l}(\phi, z)}{\alpha \delta_{m}^{2}-j \Omega}
$$

By taking the similar derivation process in [14], we can obtain the HOM content for the quantum noise (QN) induced MI from Eq. (1), which is given as

$$
\begin{aligned}
& \xi(L) \approx \frac{\hbar \omega_{0}}{P_{1}(L)} \sqrt{\frac{2 \pi}{\int_{0}^{L} P_{1}(z)\left|\chi^{\prime \prime}\left(\Omega_{0}\right)\right| d z}} \\
& \times \exp \left\{\int_{0}^{L}\left[\iint g(r, \phi, z) \psi_{2} \psi_{2} r d r d \phi\right] d z+\int_{0}^{L} P_{1}(z) \chi\left(\Omega_{0}\right) d z\right\}
\end{aligned}
$$

where $L$ is the length of the gain fiber. For the other case that MI is seeded by intensity noise, we can obtain

$\xi(L)$

$$
\begin{aligned}
& \approx \xi_{0} \exp \left[\int_{0}^{L} d z \iint g(r, \phi, z)\left(\psi_{2} \psi_{2}-\psi_{1} \psi_{1}\right) r d r d \phi-\alpha_{\text {coil }} L_{\text {coil }}\right] \\
& +\frac{\xi_{0}}{4} \sqrt{\frac{2 \pi}{\int_{0}^{L} P_{1}(z)\left|\chi^{\prime \prime}\left(\Omega_{0}\right)\right| d z}} \exp \left[\int_{0}^{L} d z \iint g(r, \phi, z)\left(\psi_{2} \psi_{2}-\psi_{1} \psi_{1}\right) r d r d \phi+\int_{0}^{L} P_{1}(z) \chi\left(\Omega_{0}\right) d z-\alpha_{\text {coil }} L_{\text {coil }}\right] R_{N}\left(\Omega_{0}\right)
\end{aligned}
$$

where $R_{N}(\Omega)$ is the relative intensity noise (RIN) of the input signal, $\xi_{0}$ is the initial frequency shifted HOM content, $\alpha_{\text {coil }}$ is the bend-induced power loss by fiber coiling, and $L_{\text {coil }}$ is the length of the coiled length. Here bend induced loss is taken into consideration in a simple way, and the effect of bend induced mode distortion has not been considered.

\subsection{Numerical results}

In this section, we have calculated the MI threshold power of the amplifier based on the model. The parameters of the fiber is the same as those in the experiment, which are listed in Table 1.

Table 1. Parameters of Test Amplifier

\begin{tabular}{cc}
\hline$R_{\text {core }}$ & $10.5 \mu \mathrm{m}$ \\
$R$ & $200 \mu \mathrm{m}$ \\
$n_{0}$ & 1.45146 \\
$N A$ & 0.064 \\
$\lambda_{\mathrm{p}}$ & $915 \mathrm{~nm}$ \\
$\lambda_{\mathrm{s}}$ & $1064 \mathrm{~nm}$ \\
$h_{\mathrm{q}}$ & $5000 \mathrm{~W} /\left(\mathrm{m}^{2} \mathrm{~K}\right)$ \\
$\eta$ & $1.2 \times 10^{-5} \mathrm{~K}^{-1}$ \\
$\kappa$ & $1.38 \mathrm{~W} /(\mathrm{Km})$ \\
$\rho C$ & $1.54 \times 10^{6} \mathrm{~J} /\left(\mathrm{Km}^{3}\right)$ \\
$\sigma_{\mathrm{p}}{ }^{\mathrm{a}}$ & $6.04 \times 10^{-25} \mathrm{~m}^{2}$ \\
$\sigma_{\mathrm{p}}{ }^{\mathrm{e}}$ & $1.96 \times 10^{-26} \mathrm{~m}^{2}$ \\
$\sigma_{\mathrm{s}}{ }^{\mathrm{a}}$ & $6.0 \times 10^{-27} \mathrm{~m}^{2}$ \\
$\sigma_{\mathrm{s}}{ }^{\mathrm{e}}$ & $3.58 \times 10^{-25} \mathrm{~m}^{2}$ \\
$N_{Y b}$ & $3.5 \times 10^{25} \mathrm{~m}^{-3}$ \\
\hline
\end{tabular}

Firstly, we calculated the MI threshold power of the amplifier. Figure 4 (a) shows the HOM content versus the pump power. It is shown that the quantum-induced-MI threshold power is about $4.7 \mathrm{~kW}$. However, intensity noise of the signal (RIN $=10^{-10}$ that correspond to a laser with high RIN, which yielding a realistic MI threshold $[23,24])$ reduces the threshold to be about $2 \mathrm{~kW}$, which agrees well with the experimental results. The initial HOM is set to be 
0.01 . By reducing the intensity noise of the signal $\left(\mathrm{RIN}=10^{-11}\right)$, the $\mathrm{MI}$ threshold power can be increased about $\sim 300 \mathrm{~W}$, which means that measures taken to reduce the intensity noise of the input signal result in only modest improvements in the MI threshold and adding to the overall complexity of the system [25]. The influence of HOM power was also calculated in Fig. 4 (b), which shows that the efforts to optimize the in-coupling of the signal have little impact on the MI threshold, which agrees with the experimental results [9].

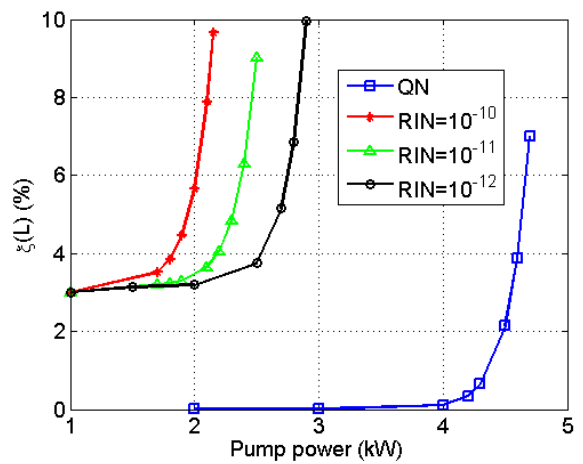

(a)

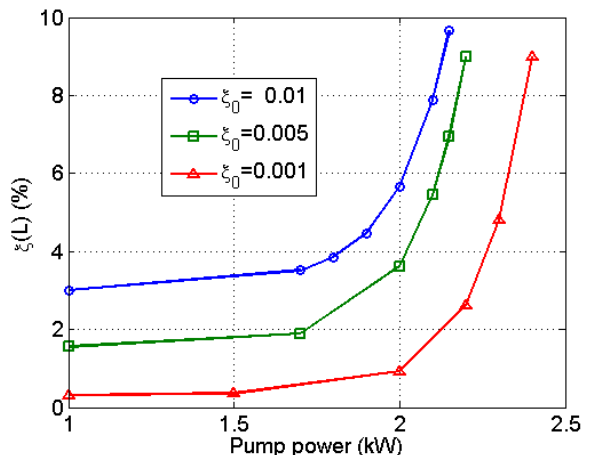

(b)

Fig. 4 Threshold calculation of the fiber amplifier.

It is reported in [26] that MI threshold power can be increased obviously by increasing cladding diameters. This is contradictive to those reported in [7], which shows that the improvement by increasing cladding diameter was not large enough for significant power scaling. To study the effect of cladding diameter on MI threshold power, we calculated the MI threshold pump power for different cladding diameters, which is shown in Fig. 5. The computed thresholds are seen to rise with increasing cladding diameters and the resulting increasing degree of population saturation [26]. However, for fiber with larger core diameters, the improvement becomes less significant, which explained the experimental results in [26]. Although MI threshold power can be improved by increasing cladding diameter, a longer length is required for fibers with larger cladding diameter to maintain good amplifier efficiency, which makes nonlinear effect suppression more challenging.

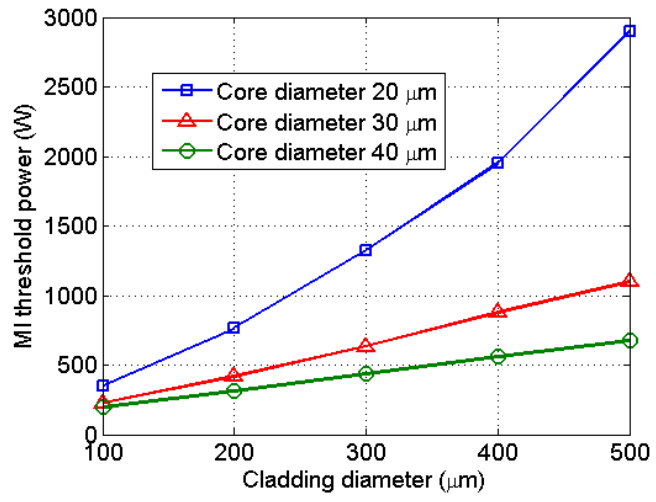

Fig. 5 Threshold pump power for different fiber cladding diameters.

Coiling the fiber with a diameter small enough to induce mode-dependent bend losses can suppress high order mode effectively [27], which can improve the MI threshold [17, 28]. The threshold power of the amplifier with tight coiling was calculated in Fig. 6. Bend losses for $\mathrm{LP}_{11}$ mode are calculated using the method of Marcuse [29]: the bending loss is $2.4 \mathrm{~dB} / \mathrm{m}$ for bending radius of $6.5 \mathrm{~cm}, 6 \mathrm{~dB} / \mathrm{m}$ for $6 \mathrm{~cm}$ and $14 \mathrm{~dB} / \mathrm{m}$ for $5.5 \mathrm{~cm}$. In practice, only first half of the gain fiber is coiled with small diameter, so $L_{\text {coil }}$ is set to be $6 \mathrm{~m}$. These coiling radiuses are chosen for long-term use. It shows that tight coiling of the fiber can increase the MI threshold power significantly: when coiling at the radius of $5.5 \mathrm{~cm}$, MI threshold is 3 times higher than 
that without coiling or loose coiling, which means that tight coiling of the gain fiber is an effective method to mitigate MI in all-fiber MOPA configuration with standard step-index LMA fiber. For the case in the experimental, MI is no longer a limitation since the onset of other nonlinear effects, such as SBS and/ or SRS, should be come into consideration first.

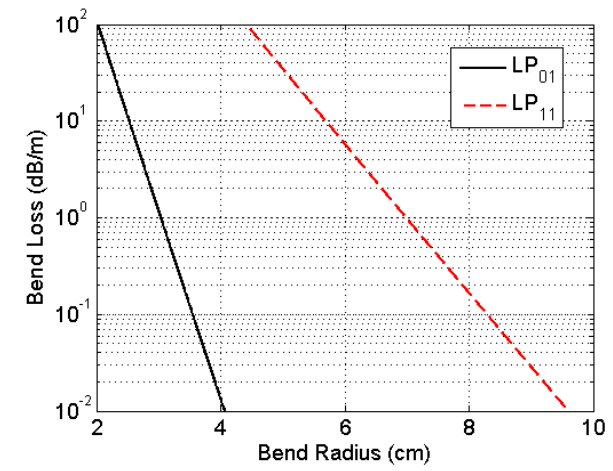

(a)

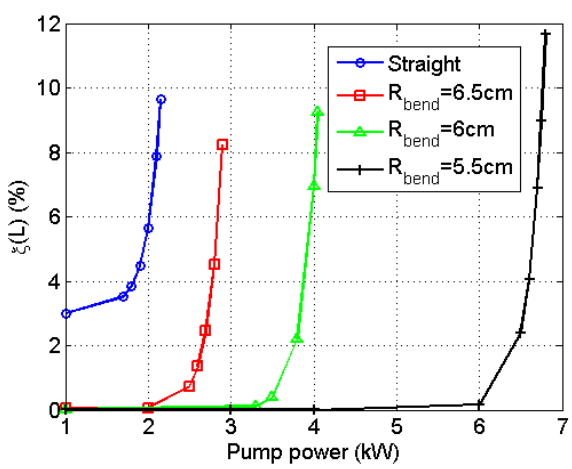

(b)

Fig. 6 The effect of coiling on MI threshold power.

\subsection{Experimental validation}

To verify our theoretical predication of the effect of coiling, we have rebuilt our fiber amplifiers with gain fiber coiled at the diameter of $\sim 12 \mathrm{~cm}$. Then we have MI-free $1280 \mathrm{~W}$ linearly-polarized single-mode laser, and all phenomenon related to the onset of MI, such as temporal fluctuation, PER degradation, have vanished (as shown in Figs. 7(a) and (b)). Due to the available pump power, the power scaling capabilities by coiling method has not been fully exploited. The advantage of employing coiling technique is that it is straightforward to implement, as most fibers are coiled in packaging anyway, and no special gain profiles are necessary to give preferential gain to fiber modes. In addition, PER of the amplifier has also been improved to $\sim 96 \%$ at the maximal operation. The results also indicate that MI can be mitigated by designing fiber with an improved delocalization of HOM, such as chirallycoupled core (CCC) fiber [30], leakage channel fibers[31], all-solid photonic bandgap fibers [32].

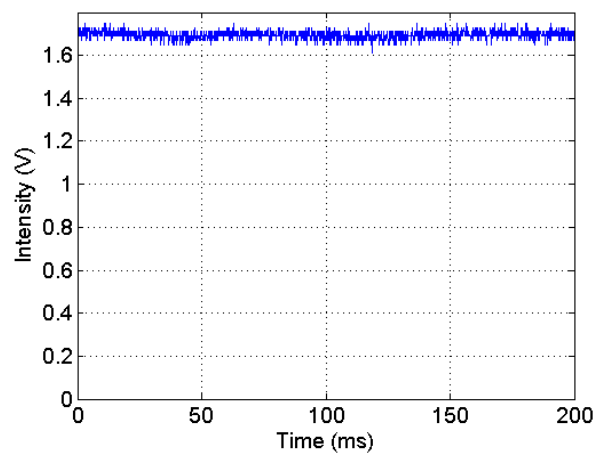

(a) Time trace

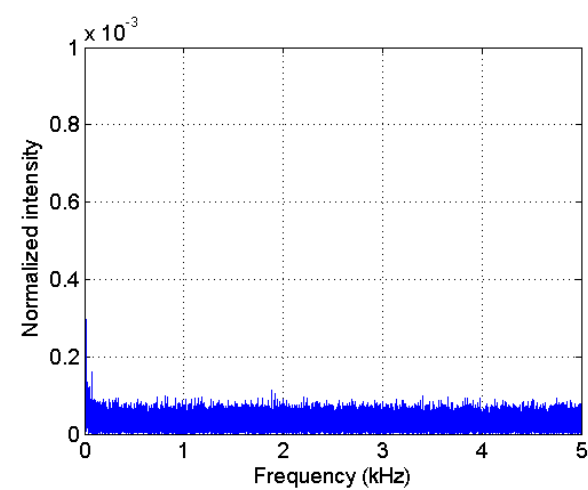

(b) Frequency distribution

Fig. 7 Fluctuation characteristics of the output beam after tight coiling.

\section{Conclusions}

We have generated a high-power linearly-polarized single-mode output laser from an $\mathrm{Yb}$ doped PM fiber amplifier, which operated at $\sim 1064 \mathrm{~nm}$. The slope efficiency of the amplifier is $65.3 \%$ with respect to launched pump power, and the maximal output power is $1261 \mathrm{~W}$. The $M^{2}$ at full power operation is measured to be $<1.2$ in both directions. The linearly-polarization 
operation was deteriorated by the onset of MI above $1.2 \mathrm{~kW}$. The PER is measured to be $>94 \%$ without MI, which reduced to about $90 \%$ after the onset of MI. A novel theoretical model to study MI has been built up, the numerical results agree well with the experimental observation. Various method to improve the power scaling capability of the amplifier without MI have been studied numerically, which reveal that MI can be suppressed by proper coiling and the amplifier in the paper have the potential to deliver MI-free $3 \mathrm{~kW}$ output power. An additional experimental has been carried out to study the effect of coiling on MI, which rebuilt the amplifier with tighter coiling. It is shown that MI was suppressed successfully in the amplifier by tight coiling.

\section{Acknowledgments}

The authors would like to acknowledge the support of Hanwei Zhang, Xiong Wang, Hailong $\mathrm{Yu}$, Baolai Yang. The research leading to these results has received funding from the program for the National Science Foundation of China under grant No. 61322505, the program for New Century Excellent Talents in University, the Innovation Foundation for Excellent Graduates in National University of Defense Technology under grant B120704 and Hunan Provincial Innovation Foundation for Postgraduate under grant CX2012B035. 